\title{
Caracterização dos fragmentos florestais amazônicos remanescentes na microbacia hidrográfica do rio Taxidermista I em Alta Floresta, MT
}

\author{
Monica Elisa Bleich ${ }^{1 *}$ \\ Carlos José da Silva ${ }^{2}$ \\ ${ }^{1}$ Universidade do Estado de Mato Grosso \\ Caixa Postal 324, Campus Universitário de Alta Floresta, CEP 78580-000, Alta Floresta - MT, Brasil \\ ${ }^{2}$ Universidade de Brasília, Brasília - DF, Brasil \\ *Autor para correspondência \\ mebleich@yahoo.com.br
}

Submetido em 19/04/2013

Aceito para publicação em 28/08/2013

\section{Resumo}

A manutenção da conectividade dos remanescentes florestais é fundamental, e a preservação da vegetação ripária dos riachos na paisagem, contribui muito com a manutenção da integridade do mosaico de habitats. $\mathrm{O}$ objetivo deste estudo foi caracterizar os fragmentos florestais amazônicos remanescentes na microbacia hidrográfica do rio Taxidermista I em Alta Floresta, MT, visando fornecer subsídios para a implantação dos projetos de recuperação das áreas degradadas e a conservação dos fragmentos florestais. Os fragmentos florestais foram vetorizados e posteriormente avaliados seu tamanho, perímetro e distância de cada fragmento a partir da borda até o curso de água mais próximo. Foi também determinado o índice de circularidade, ou da relação borda/interior. Na microbacia estudada foram registrados 63 fragmentos florestais com tamanho variando entre 0,44 e 67,51 ha e perímetro entre 262,67 e 4756,26 m. A distância dos fragmentos florestais até o curso de água mais próximo varia de 0 a $450 \mathrm{~m}$, no entanto $71,4 \%$ dos fragmentos estão conectados a um curso de água. Nos fragmentos florestais da microbacia hidrográfica do rio Taxidermista I predomina a forma alongada, apresentam área pequena, mas com grande perímetro/borda em relação ao tamanho, o que os torna suscetíveis ao efeito de borda potencializado pela matriz que abriga a atividade pecuária.

Palavras-chave: Amazônia; Fragmentação da paisagem; Manejo integrado

\section{Abstract}

Characterization of Amazonian forest fragments in the catchment of the Taxidermista I River in Alta Floresta, MT. Maintaining the connectivity of the remaining forest in the state of Mato Grosso is critically important, and the preservation of riparian vegetation of streams contributes greatly to retaining the integrity of the mosaic of habitats found in this region. The aim of this study was to characterize the Amazonian forest fragments remaining in the catchment of the Taxidermista I River, in Alta Floresta, MT, in order to provide input for the implementation of recovery projects in degraded areas and the conservation of forest fragments. The forest fragments were vectorized and the size, perimeter, and distance of each fragment to the nearest watercourse were evaluated. The circularity index, or the edge/interior ratio, was also determined. In the watershed of the Taxidermista I River, 63 forest fragments ranging in size from 0.44 to 67.51 ha, with perimeters between 262.67 
and $4756.26 \mathrm{~m}$, were recorded. The distance from each forest fragment to the nearest watercourse ranges from 0 to $450 \mathrm{~m}$; however, $71.4 \%$ of the fragments are connected to a watercourse. The majority of the fragments in this watershed are elongated and occupy a small area, but their perimeter/edge is large relative to their size, which makes them susceptible to edge effects, especially from nearby cattle farms.

Key words: Amazon; Integrated management; Landscape fragmentation

\section{Introdução}

Os fragmentos florestais podem conter os últimos vestígios de habitat intacto, fauna e outros recursos naturais em paisagens dominadas pelo homem, no entanto, o isolamento os torna cada vez mais vulneráveis (PRINGLE, 2000), visto que sua localização muitas vezes é determinada pelo desenvolvimento socioeconômico (PRINGLE, 2001). A matriz da paisagem influencia a conectividade dos fragmentos, e essa é uma das principais conclusões do projeto dinâmica biológica de fragmentos florestais (PDBFF) na Amazônia (LAURANCE et al., 2002).

Por outro lado, a integridade biológica de um fragmento florestal é afetada por alterações na conectividade hidrológica dentro e fora dos limites da bacia hidrográfica (PRINGLE, 2001). O sucesso limitado dos esforços para conservação de muitos rios deve-se à falta de compreensão do papel crítico das perturbações, processos sucessionais e interações dos elementos da paisagem, sustentando a integridade ecológica desses ecossistemas aquáticos (WARD et al., 2002).

A manutenção da conectividade dos remanescentes florestais é fundamental, e a preservação da vegetação ripária dos riachos na paisagem, contribui muito com a manutenção da integridade do mosaico de habitats em uma determinada região (PRINGLE, 2001; LAURANCE et al., 2002). A delimitação do tamanho e forma de fragmentos florestais, bem como o conhecimento da distância entre os remanescentes florestais e a vegetação ripária são parâmetros úteis (VALENTE; VETTORAZZI, 2002) e podem subsidiar o planejamento de ações de recuperação de áreas de reserva legal. Da mesma forma contribuindo para a manutenção das áreas de preservação permanente nas propriedades rurais conectando-as aos corredores ripários de toda a paisagem.
Ações para recuperação das áreas degradadas na microbacia hidrográfica Taxidermista I, promovidas pela Secretaria Municipal de Meio Ambiente de Alta Floresta - MT, estão em andamento, no entanto ainda faltam informações acerca dos remanescentes florestais, como sua forma, tamanho, e conectividade na paisagem da microbacia hidrográfica. Estas informações podem ser úteis para um melhor planejamento do uso e recuperação das áreas degradadas na microbacia hidrográfica.

Neste sentido, a utilização do Sistema de Informação Geográfica (SIG) é fundamental, visto que o planejamento da conservação de fragmentos florestais é facilitada por esta ferramenta. O SIG pode ser útil na definição, implementação e fiscalização das áreas de florestas ripárias destinadas à conservação. De acordo com Sartori et al. (2012), as informações geoespaciais sobre o meio biofísico têm potencial para favorecer a conexão entre os fragmentos florestais, além de servir como forte instrumento de orientação às políticas públicas e aos processos coletivos de decisão.

O objetivo desse estudo foi caracterizar a estrutura espacial dos fragmentos florestais amazônicos remanescentes na microbacia hidrográfica do rio Taxidermista I em Alta Floresta, MT, visando fornecer subsídios para a implantação dos projetos de recuperação das áreas degradadas e o manejo conservacionista dos fragmentos florestais.

\section{Material e Métodos}

O estudo foi desenvolvido na microbacia hidrográfica do rio Taxidermista I, também conhecida como microbacia Mariana, localizada no município de Alta Floresta, região norte do estado de Mato Grosso (Figura 1). A cidade de Alta Floresta está a $283 \mathrm{~m}$ de altitude, o clima é equatorial quente e úmido e a temperatura média anual é de $24^{\circ} \mathrm{C}$, com 
FIGURA 1: Localização dos fragmentos florestais na microbacia hidrográfica do rio Taxidermista I em Alta Floresta, MT.

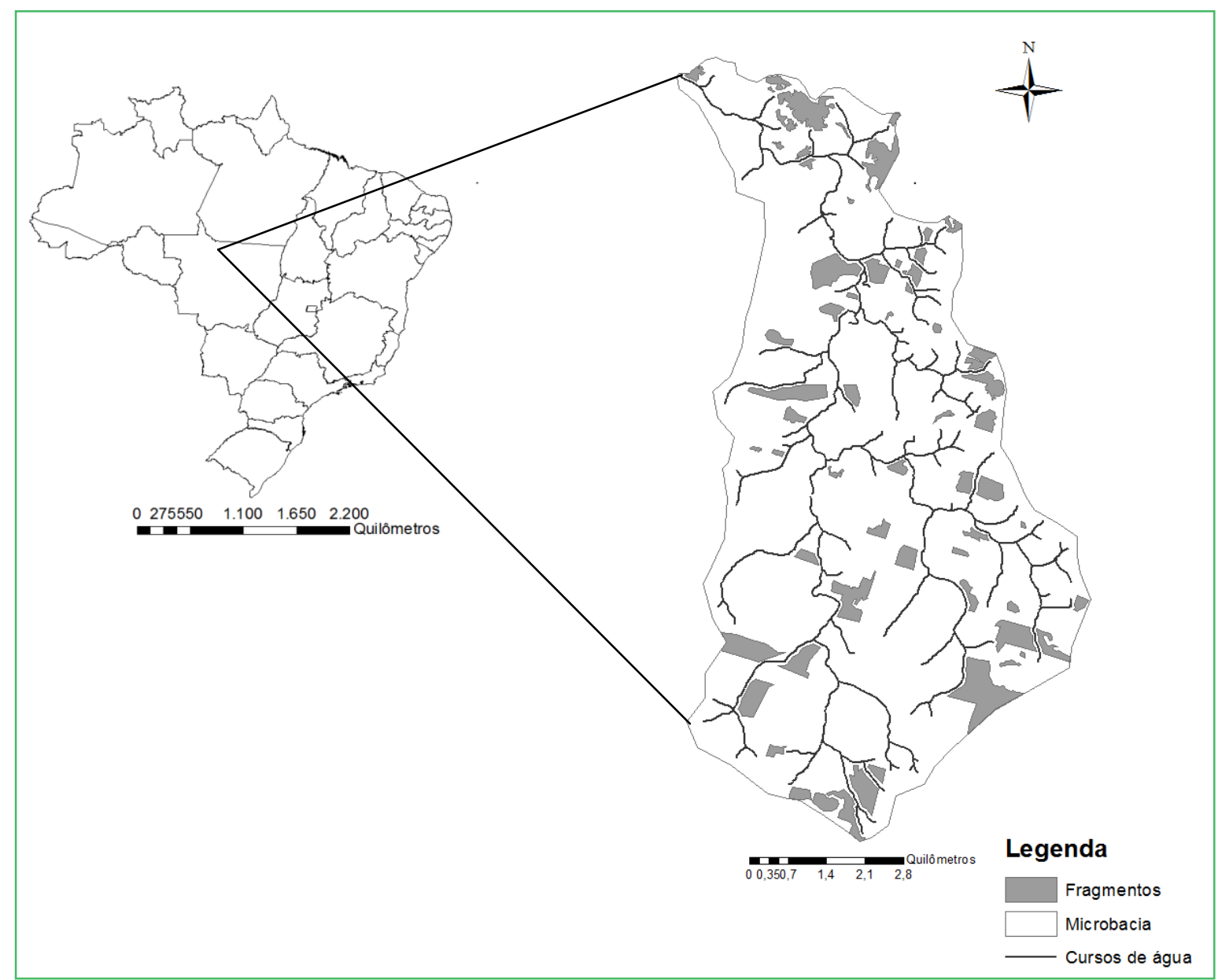

máxima de $40^{\circ} \mathrm{C}$. O município compreende um total de $8.947,00 \mathrm{~km}^{2}$ de área territorial e uma população estimada de 49.117,00 habitantes (IBGE, 2007); e esta ocupação humana vem comprometendo a viabilidade dos recursos hídricos tanto em áreas urbanas como rurais. Na região, predomina a vegetação do tipo Floresta Ombrófila Aberta (IBGE, 2012), latossolos, solos podzólicos, solos litólicos distróficos, solos indiscriminados concrecionários e ao longo dos rios ocorrem solos do tipo gley pouco húmico, e areias quartzosas (SEPLAN, 2000). Atualmente, a pecuária é principal atividade econômica desenvolvida nesta microbacia hidrográfica (BLEICH et al., 2012). O diagnóstico ambiental realizado para a Agenda 21 Local de Alta Floresta mostrou que cerca de $70 \%$ da microbacia hidrográfica do rio Taxidermista I encontrase degradada (MICOL et al., 2008).
A microbacia hidrográfica do rio Taxidermista I pertence à sub-bacia do rio Teles Pires, afluente do rio Tapajós, e é a fonte de abastecimento de água para a cidade de Alta Floresta. A microbacia está localizada entre as latitudes $9^{\circ} 52^{\prime} 0$ " e $10^{\circ} 0$ '0"S e longitudes $56^{\circ} 9^{\prime} 0$ " e $56^{\circ} 6^{\prime} 0$ "O do município de Alta Floresta. A microbacia compreende uma área de 6.501 ha, dentre os quais 991 ha são áreas de preservação permanente (APP) hídrica e abrigam 62 nascentes hidrográficas. Esta microbacia foi apontada como área prioritária para a recuperação no município ao longo dos trabalhos desenvolvidos pela Agenda 21 Local, uma vez que apenas $43 \%$ das APPs hídricas apresentam cobertura florestal (MICOL et al., 2008).

A caracterização dos fragmentos foi feita utilizando imagens do satélite SPOT-5 (Satellite Probatoire pour l'Observation de La Terre), com resolução espacial 
de 2,5 m, do ano de 2009, composição colorida RGB, georreferenciadas, obtidas junto à Secretaria do Meio Ambiente (SEMA) do Mato Grosso. Foi utilizado um arquivo vetorial da rede hidrográfica da microbacia do rio Taxidermista I elaborado pelo Instituto Centro de Vida (ICV), o qual subsidiou o Cadastro Ambiental Rural (CAR) das propriedades desta microbacia. Todos os fragmentos florestais da microbacia Taxidermista I foram vetorizados no programa ArcGis 9.3 (ESRI, 2006), nas coordenadas projetadas UTM Zona 21 Sul, sistema de Referência Geográfica SAD69, e foram avaliados em relação a área (ha) e ao perímetro (m). Também foi estimada a distância $(\mathrm{m})$ de cada fragmento a partir da borda até o curso de água mais próximo.

O índice de circularidade (IC), ou da relação borda/ interior, foi determinado com base em Chaturvedi (1926, apud OLIVEIRA et al., 2005), Greggio et al. (2009) e também por Costa (2011), para propor a tendência em relação à forma dos fragmentos, conforme a fórmula:

$$
\mathrm{IC}=\frac{40000 \cdot \pi \cdot \mathrm{A}}{\mathrm{P}^{2}}
$$

Sendo: $\mathrm{IC}=$ Índice de circularidade; $\mathrm{A}=$ Área do fragmento (ha); $\mathrm{P}=$ Perímetro do fragmento (m).

Segundo o IC, o fragmento apresenta tendência à forma arredondada quando o valor for próximo de 1 ( $\mathrm{IC}=1$, perfeitamente circular), e a forma alongada quando os valores se aproximam de $0(\mathrm{IC}=0$, alongado).

Os dados foram analisados no pacote BiodiversityR, implementado na plataforma R (R DEVELOPMENT CORE TEAM, 2011), quanto aos valores máximos e mínimos, média e desvio padrão para o tamanho e perímetro, frequência de ocorrência dos fragmentos com relação às categorias do índice de circularidade (IC $<0,60$; IC entre 0,61 e 0,85 e IC $>0,85)$. A normalidade na distribuição dos dados foi testada pela função Shapiro do pacote Stats, e após verificado que os dados não apresentam distribuição normal foi testada a correlação de Spearman entre área e o perímetro dos fragmentos, e a correlação de Spearman entre a área dos fragmentos, ou o perímetro, e a distância do fragmento em relação ao curso de água mais próximo.

\section{Resultados e Discussão}

Foram registrados 63 fragmentos florestais (Figura 1), com tamanho variando entre 0,44 e 67,51 ha (média \pm desvio padrão: $10,12 \pm 12,15$ ) e perímetro entre 262,67 e $4.756,26 \mathrm{~m}(1.464,80 \pm 1.048,71)$. Os fragmentos florestais na microbacia estudada totalizam uma área de 637,45 ha. A distância dos fragmentos florestais até o curso de água mais próximo varia de 0 a $450 \mathrm{~m}(52,06 \pm 106,67)$, no entanto $71,4 \%$ dos fragmentos estão conectados a um curso de água.

Fragmentos com área inferior a 5 ha somam $46 \%$ na paisagem da microbacia estudada e são em média os fragmentos mais distantes dos cursos de água. Por outro lado, os fragmentos com área maior que $30 \mathrm{~h}$ estão conectados aos cursos de água da microbacia (Tabela 1). A distância dos fragmentos em relação ao curso de água mais próximo apresentou correlação significativa negativa com a área dos fragmentos $\left(r_{s}=-0,45 ; p<0,01\right)$, e também com o perímetro dos fragmentos $\left(r_{s}=-0,44 ; p<0,01\right)$, já a área e o perímetro dos fragmentos florestais da microbacia apresentaram correlação significativa positiva $\left(r_{s}=0,95 ; p<0,01\right)$. Alto percentual de fragmentos de tamanho reduzido, com área

TABELA 1: Características dos fragmentos florestais da microbacia hidrográfica do rio Taxidermista I em Alta Floresta, MT.

\begin{tabular}{lccccccccc}
\multicolumn{2}{c}{ Fragmentos } & \multicolumn{2}{c}{ Área } & \multicolumn{2}{c}{ Perímetro } & \multicolumn{2}{c}{ IC } & \multicolumn{2}{c}{ Distância } \\
\hline \multicolumn{1}{c}{ Categoria } & $\%$ & Média & $\begin{array}{c}\text { Desvio } \\
\text { Padrão }\end{array}$ & Média & $\begin{array}{c}\text { Desvio } \\
\text { Padrão }\end{array}$ & Média & $\begin{array}{c}\text { Desvio } \\
\text { Padrão }\end{array}$ & Média & $\begin{array}{c}\text { Desvio } \\
\text { Padrão }\end{array}$ \\
\hline$<5 \mathrm{ha}$ & 46 & 2,31 & 1,39 & 735,68 & 307,52 & 0,56 & 0,19 & 88,45 & 136,08 \\
$>5 \mathrm{e}<10 \mathrm{~h}$ & 22 & 6,70 & 1,17 & $1.266,32$ & 192,89 & 0,55 & 0,14 & 36,79 & 87,78 \\
$>10 \mathrm{e}<30 \mathrm{ha}$ & 22 & 17,26 & 6,25 & $2.143,76$ & 629,92 & 0,53 & 0,22 & 14,29 & 39,56 \\
$>30 \mathrm{ha}$ & 10 & 39,19 & 14,17 & $3.867,83$ & 886,28 & 0,36 & 0,15 & 0,00 & 0,00 \\
\hline
\end{tabular}


menor que 5 ha, foi também registrado por Martins et al. (2002), no estado do Tocantins, e por Vidolin et al. (2011), no estado do Paraná.

O índice de circularidade (IC), ou da relação borda/interior, foi determinado para propor a tendência em relação à forma de um fragmento (COSTA, 2011). O IC registrado para os fragmentos da microbacia hidrográfica do rio Taxidermista I variou de 0,19 a $0,89(0,53 \pm 0,19)$, sendo que $62 \%$ dos fragmentos apresentaram IC menor que 0,60 , enquanto que $36,5 \%$ apresentaram IC entre 0,65 e 0,85 e apenas um fragmento apresentou IC $>0,85$. Logo, predominaram na microbacia estudada fragmentos com tendência a forma alongada e recortada (Tabela 1). Índices de circularidade entre 0,20 e 0,60 indicam que os fragmentos têm formato bastante recortado, mesmo no caso de fragmentos extensos, e valores de IC entre 0,65 e 0,85 demonstram uma tendência moderadamente alongada (LOPES et al., 2011; ANDRADE et al., 2012). Fragmentos alongados e recortados terão maior perímetro (ou borda) e, provavelmente, serão os mais afetados pelo efeito de borda (OLIFIERS; CERQUEIRA, 2006), que modifica as condições ambientais no interior do fragmento (KAPOS, 1989) devido à modificação da estrutura da floresta próximo da borda (KAPOS et al., 1997). No entanto, as espécies podem responder de forma distinta ao mesmo gradiente de condições ambientais (KAPOS et al., 1997).

Primack e Rodrigues (2001) discutem um exemplo hipotético de que um fragmento quadrado com 100 ha teria $4.000 \mathrm{~m}$ de perímetro, e se for considerado que a luz que incide lateralmente na floresta penetre $100 \mathrm{~m}$ a partir da borda da floresta, restariam apenas 64 ha no interior do fragmento com as condições originais e as espécies típicas conservadas. Neste estudo, os fragmentos apresentaram perímetro superior ao que corresponderia a uma forma quadrada ou retangular sem recortes, a exemplo o fragmento com área de 67,51 ha e que apresentou perímetro superior a uma área de 100 ha. Isso indica que, além das formas não serem circulares, ainda há uma grande borda que favorecerá a alteração das condições ambientais, aumento da temperatura e redução da umidade próximo da borda do fragmento (KAPOS, 1989), o que dificultaria a conservação da diversidade biológica nestes fragmentos amazônicos sem um manejo adequado.

Segundo Greggio et al. (2009), a importância da análise de IC para os estudos da dinâmica e estrutura dos fragmentos florestais evidencia-se na possibilidade de indicar o nível de proteção de seu interior em relação aos efeitos de borda. Os fragmentos estudados apresentam um baixo índice de circularidade o que implica em uma alta suscetibilidade ao efeito de borda e, consequentemente, estão sujeitos a um maior grau de perturbação. De acordo com Primack e Rodrigues (2001), áreas com forma circular minimizam a relação borda-área, distanciando o centro dos fragmentos de qualquer borda, ao contrário das formas alongadas. A borda é a região do fragmento sob maior influência da matriz da paisagem (LOPES et al., 2011), logo quanto mais alongado for o fragmento maior será a área exposta aos efeitos resultantes de ações antrópicas (MARTINS et al., 2002).

Lopes et al. (2011) classificaram o tamanho dos fragmentos na APA Pratigi, baixo sul da Bahia, e, baseados nos estudos de Laurence et al. (1997) e de Valente e Vettorazzi (2002), afirmaram existir uma relação importante entre a área e o valor do fragmento para a conservação da biodiversidade. De acordo com esta classificação, fragmentos com alto valor apresentam medidas maiores que 300 ha, fragmentos com valor mediano apresentam entre 3 e 300 ha e fragmentos com valor baixo apresentam medidas menores que 3 ha. Logo, nenhum fragmento analisado aqui seria classificado como de alto valor para a conservação da biodiversidade, uma vez que apresentaram área entre 0,44 a 67,51 ha. No entanto, considerando que a paisagem da microbacia em estudo encontra-se muito fragmentada e inexistem áreas próximas de floresta contínua, a conservação de fragmentos florestais com área inferior a 70 ha torna-se de alto valor para a conservação da biota nesta região da Amazônia, mesmo que não mantenham ótimas condições e recursos a todas as espécies da região. Isso implica em destinar maior atenção aos fragmentos da microbacia hidrográfica do rio Taxidermista I, para que não deixem de oferecer os serviços ambientais como a polinização, a dispersão e fonte de propágulos, e enfatiza a necessidade de melhorar as condições internas 
e externas destes fragmentos, bem como conectá-los a outras áreas conservadas próximas, e assim ter na paisagem fragmentos maiores e mais biodiversos.

Corredores ripários podem ajudar a manter um nível de conectividade em paisagens florestais fragmentadas (LIMA; GASCON, 1999). Considerando as distâncias existentes entre os fragmentos florestais e os cursos de água, que não ultrapassam $450 \mathrm{~m}$, seria possível que um bom planejamento integrado da recuperação (revegetação) das áreas degradadas restabeleça a conectividade entre os fragmentos por meio da vegetação ripária que atuaria como corredor para os fragmentos remanescentes na microbacia. A conexão entre os fragmentos aumentaria a área de forrageamento para animais, o que também poderia minimizar os efeitos causados pelo pastoreio dos animais silvestres nestas áreas.

A partir dos resultados, é possível concluir que entre os fragmentos florestais da microbacia hidrográfica do rio Taxidermista I predomina a forma alongada, apresentam área pequena, mas com grande perímetro/ borda em relação ao tamanho, o que os torna suscetíveis ao efeito de borda potencializado pela matriz da paisagem que abriga, predominantemente, a atividade pecuária. A fragmentação causa alterações importantes na dinâmica das florestas amazônicas, especialmente nos primeiros $100 \mathrm{~m}$ da borda dos fragmentos, e esse efeito de borda altera a estrutura da floresta, sendo agravados os efeitos em espécies sensíveis às perturbações (LAURENCE et al., 1998). Entretanto, corredores ligando os fragmentos podem reduzir os impactos da fragmentação florestal (LAURANCE; LAURANCE, 2000). Apesar de serem poucas e pequenas as áreas florestais remanescentes na microbacia estudada, sua conservação é imprescindível bem como a integração entre os remanescentes, principalmente por meio da conexão às APPs hídricas.

Remanescentes florestais podem atuar como catalisadores importantes para a regeneração da floresta, então fragmentos pequenos precisam ser conectados por corredores florestais a áreas de floresta. As florestas plantadas para a colheita de frutas ou outros produtos florestais podem proteger as bordas dos fragmentos florestais, além de contribuir para a dispersão de espécies entre áreas conservadas (BIERREGAARD JR. et al.,
1992). Além disso, a conectividade dos fragmentos a floresta ripária pode facilitar o manejo integrado dessas áreas a fim de melhorar a estrutura e o funcionamento do sistema. Assim, ressalta-se a necessidade de programar estratégias de manejo conservacionista para os fragmentos florestais, visando minimizar os riscos de deterioração e extinção destes fragmentos. A recuperação da floresta ripária aumentará a conectividade dos fragmentos florestais e é apontada como uma alternativa para a manutenção da biodiversidade.

\section{Referências}

ANDRADE, L. S; SILVA, E. N.; RIBEIRO, G. A; PARO, S. P.; PAULA, M. O. Avaliação de fragmentos florestais em uma região do Quadrilátero ferrífero: Municípios de Mariana e Ouro Preto. Enciclopédia Biosfera, Goiânia, v. 8, n. 14, p. 10-51, 2012.

BIERREGAARD JR., R. O.; LOVEJOY, T. E.; KAPOS, V.; DOS SANTOS, A. A.; HUTCHINGS, R. W. The biological dynamics of tropical rainforest fragments: a prospective comparison of fragments and continuous forest. BioScience, Berkeley, v. 42, n. 11, p. 859-866, 1992.

BLEICH, M. E.; ROBOREDO, D.; SILVA, E. P.; BERGAMASCO, S. M. P. P. Caracterização limnológica de riachos de cabeceira em Alta Floresta-MT: subsídios para avaliação da eficácia de ações de recuperação de áreas ripárias degradadas. In: SIMPÓSIO PROCESSOS ECOLÓGICOS, RESTAURAÇÃO E ECOVALORAÇÃO EM ZONAS RIPÁRIAS, I, 2012, Brasília. Anais... Brasília: AQUARIPÁRIA/UnB/EMBRAPA, 2012. p. 35. Versão eletrônica.

COSTA, C. C. Subsídios para a proteção dos fragmentos florestais na bacia hidrográfica do rio Poxim - SE. 2011. 112 f. Dissertação (Mestrado em Agroecossistemas) - Universidade Federal de Sergipe, São Cristóvão. 2011.

ESRI - ENVIRONMENTAL SYSTEMS RESEARCH INSTITUTE. ArcGIS Professional GIS for the desktop, versão $9.3,2006$

GREGGIO, T. C.; PISSARRA, T. C. T.; RODRIGUES, F. M. Avaliação dos fragmentos florestais do município de JaboticabalSP. Revista Árvore, Viçosa, v. 33, n. 1, p. 117-124, 2009.

IBGE - INSTITUTO DE GEOGRAFIA E ESTATÍSTICA. Manual técnico da vegetação brasileira. 2. ed. Rio de Janeiro: Departamento de Recursos Naturais e Estudos Ambientais/IBGE, 2012. 271 p.

KAPOS, V. Effects of isolation on the water status of forest patches in Brazilian Amazon. Journal of Tropical Ecology, Winchelsea, v. 5, p. 173-185, 1989.

KAPOS, V.; WANDELLI, E.; CAMARGO, J. L.; GANADE, G. Edge-related changes in environment and plant responses due to forest fragmentation in Central Amazonia. In: LAURENCE, W. F.; BIERREGAARD, R. O. (Ed.). Tropical forest remnants - Ecology, management and conservation of fragmented communities. Chicago: University Press, Chicago, 1997. p. 33-44. 
LAURENCE, W.; BIERREGAARD JR, R. O.; GASCON, C.; DIDHAM, R. K.; SMITH, A. P.; LYNAM, A. J.; VIANA, V. M.; LOVEJOY, T. E.; SIEVING, K. E.; SITES JR, J. W.; ANDERSEN, M.; TOCKER, M. D.; KRAMER, E. A.; RESTREPO, C.; MORITZ, C. Tropical forest fragmentation - Synthesis of a diverse and dynamic discipline. In: LAURENCE, W. F.; BIERREGAARD, R. O. (Ed.). Tropical forest remnants - Ecology, management and conservation of fragmented communities. Chicago: University Press, Chicago, 1997. p. 502-514.

LAURANCE, W. F.; FERREIRA, L. V.; RANKIN-DE MERONA, J. M.; LAURANCE, S. G. Rainforest fragmentation and the dynamics of Amazonian tree communities. Ecology, Washington, v. 79, n. 6, p. 2032-2040, 1998.

LAURANCE, S. G.; LAURANCE, W. F. Tropical corridors: use of linear rainforest remnants by arboreal mammals. Biological Conservation, Philadelphia, v. 91, p. 231-240, 2000.

LAURANCE, W. F.; LOVEJOY, T. E.; VASCONCELOS, H. L.; BRUNA, E. M.; DIDHAM, R. K.; STOUFFER, P. C.; GASCON, C.; BIERREGAARD, R. O.; LAURANCE, S. G.; SAMPAIO, E. Ecosystem decay of Amazonian forest fragments: a 22-year investigation. Conservation Biology, Philadelphia, v. 16, n. 3, p. 605-618, 2002.

LOPES, N. S.; MOREAU, M. S.; MORAES, M. E. B. Análise da paisagem com base na Fragmentação - caso APA Pratigi, baixo sul da Bahia, Brasil. REDE - Revista Eletrônica do Prodema, Fortaleza, v. 6, n. 1, p. 53-67, 2011.

LIMA, M. G.; GASCON, C. The conservation value of linear forest remnants in central Amazonia. Biological Conservation, Philadelphia, v. 91, p. 241-247, 1999.

MARTINS, I. C. M.; SOARES, V. P.; SILVA, E.; BRITES, R. S. Diagnóstico ambiental no contexto da paisagem de fragmentos florestais naturais "Ipucas" no município de Lagoa da Confusão, Tocantins. Revista Árvore, Viçosa, v. 26, n. 3, p. 299-309, 2002.

MICOL, L.; ABAD, R.; BERNASCONI, P. Diagnóstico ambiental do município de Alta Floresta - MT. Cuiabá: Instituto Centro de Vida (ICV), 2008. 9 p. (Relatório Técnico)
OLIFIERS, N.; CERQUEIRA, R. Fragmentação do habitat: efeitos históricos e ecológicos. In: ROCHA, C. F. D.; BERGALLO, H. G.; VAN SLUYS, M.; ALVES, M. A. S. (Ed.). Biologia da conservação: essências. São Carlos: RiMa, 2006. p. 261-279.

OLIVEIRA, M. L. R.; SOARES, C. P. B.; SOUZA, A. L.; LEITE, H. G. Equações de volume de povoamento para fragmentos florestais naturais do município de Viçosa, Minas Gerais. Revista Árvore, Viçosa, v. 29, n. 2, p. 213-225, 2005.

PRIMACK, R. B.; RODRIGUES, E. Biologia da Conservação. Londrina: E. Rodrigues, 2001. 328 p.

PRINGLE, C. M. Riverine connectivity: conservation and management implications for remnant natural areas in complex landscapes. Plenary Talk. Verhandlungen Internationale Vereinigung Limnologie, Stuttgart, v. 27, p. 1-16, 2000.

PRINGLE, C. M. Hydrologic connectivity and the management of biological reserves: a global perspective. Ecological Applications, Washington, v. 11, n. 4, p. 981-998, 2001.

R Development Core Team. R: A language and environment for statistical computing. Vienna: R Foundation for Statistical Computing, ISBN 3-900051-07-0, URL http://www.R-project.org/. 2011.

SARTORI, A. A. C.; SILVA, R. F. B.; ZIMBACK, C. R. L. Combinação linear ponderada na definição de áreas prioritárias à conectividade entre fragmentos florestais em ambiente SIG. Revista Árvore, Viçosa, v. 36, n. 6, p. 1079-1090, 2012.

SEPLAN. Zoneamento sócio-econômico-ecológico do Estado de Mato Grosso. Cuiabá: Secretaria do Planejamento, 2000. 300 p.

VALENTE, R. O. A.; VETTORAZZI, C. A. Análise da estrutura da paisagem Bacia do Rio Corumbataí, SP. Scientia Forestalis, Piracicaba, n. 62, p. 114-129, 2002.

VIDOLIN, G. P.; BIONDI, D.; WANDEMBRUCK, A. Análise da estrutura da paisagem de um remanescente de floresta com araucária, Paraná, Brasil. Revista Árvore, Viçosa, v. 35, n. 3, p. 515-525, 2011

WARD, J. V.; MALARD, F.; TOCKNER, K. Landscape ecology: a framework for integrating pattern and process in river corridors Landscape Ecology, Oxford, v. 17, n. 10, p. 35-45. 2002. 